\title{
Bioinformatics Image Based Decision Support System for Bone Cancer Detection
}

\author{
Sravanthi Vallabojuํㅜ , P. W. C. Prasad ${ }^{1 *}$, Abeer Alsadoon ${ }^{1}$, Manoranjan Paul $^{1}$, Amr Elchouemi $^{2}$ \\ ${ }^{1}$ School of Computing and Mathematics, Charles Sturt University, Australia. \\ 2 Walden University, USA. \\ * Corresponding author. Tel.: 61292919356; email: cwithana@studygroup.com \\ Manuscript submitted August 10, 2017; accepted December 8, 2017. \\ doi: $10.17706 /$ jcp.13.7.771-783
}

\begin{abstract}
Bone cancer which may occur inside or on the bone can be life threatening similar to the other types of cancer. The aim of this paper is to improve the accuracy of the detection process. Currently, the detection process is carried out utilising data mining techniques and image processing methods as part of a medical image analysis process, using a non-automated framework which includes image acquisition, image filtering, image segmentation, the area of interest (intensity of the background or the segmented slices) and classification methods to evaluate the decision. Although these methods are effective to some extent, the existing methods have some limitations through false detection values, an increase in the processing time and accuracy. The result indicates that by using eigenvalues and eigenvectors, the processing time can be decreased by implementing normalization, while improving detection accuracy. This paper investigates the viability of using texture based magnetic resonance imaging (MRI) to locate different clusters and classify areas for determining bone cancer. This segmentation and classification processes are carried out by using eigenvalues and eigenvectors.
\end{abstract}

Key words: Eigenvectors, affinity matrix, clusters, feature extraction, normalized eigenvectors, bone tumour.

\section{Introduction}

Bone cancer is a disease that may occur inside or on a bone. Bone cancer usually involves a tumour which will be filled with abnormal cells that occasionally appear on the exterior of the bone. Bone cancer can be life threatening similar to the other types of cancer. Doctors are continuing the search for causes of cancer which are at present not fully understood. There are five various kinds of bone tumours affecting people ages between 10 and 60, all of which can occur anywhere in the body. Unfortunately, symptoms of bone cancer are not always obvious with pain being the most apparent symptom in many instances. If pain occurs, it is critical to identify the cancer as soon as possible.

In recent studies, cancer has been quoted as a heterogeneous disease which consists of different subtypes. To facilitate the subsequent clinical management of patients, early detection and projection of cancer and type of cancer are necessary in cancer research. The machine learning methods classify cancer patients into high or low-risk groups [1], [2]. Therefore, the main aim of utilizing these techniques is to diagnose cancer and increase the efficiency of treatment and progress the speed of modelling of cancerous conditions. Furthermore, the machine language tools reveal their importance by detecting the key features from complex datasets. There are various types of techniques which include Support Vector Machines (SVMs) and Decision Trees (DTs) [3]. 
Approaches to the detection of bone cancer differ widely, although some seem to have limitations in terms of effectiveness and accuracy of detection while others struggle with time constraints and false detection values [4]. In terms of speed of detection, Zhai , Huang, He, \& Liu [5] proposed a fully automated framework for linear feature selection in texture analysis using Partial Least Square (PLS). Avula, Narasimha and Murali [6] found a solution to detect the bone cancer by achieving accuracy up to 95\%, although this held true only for the region of interest (ROI) and the extracted features. If the classification is not optimal, it might produce false results and the iterations cause the delay if the given datasets are huge. Classification techniques presented in different methodologies thus have some limitations in terms of false-positive and false-negative.

Avula, Lakkakula, \& Raja [6], and Chuah, Reeth, Sheah, \& Poh [7] have proposed new techniques for dealing with of accuracy in detection. Urdaneta and Wahid [8] have proposed a method for bone tumour detection using UWB (Ultra Wideband) imaging and simulation to deal with the false detection values. The limitation of this proposal arises because of the time consuming data collection. Avula, Lakkakula, \& Raja [6] have used k-means and achieved higher accuracy levels [9]. However, the results depend upon the ROI and the extracted features. If the classification is not optimal, it might produce false results and the iterations may cause delay in producing results by producing massive datasets. The significance of the study lies in the fact that it is incorporating eigenvectors into the bone cancer detection process, also extending this process to segmentation in diagnosis of the bone cancer as it is evident that the Machine Learning techniques result in effective and accurate decision-making to solve the discussed issues.

\section{Related Work}

Based on the research explored so far, the general approach for detecting bone cancer is depicted in Fig. 1. This figure shows the different stages in the bone cancer detection process and the different techniques or methodologies implemented for each. In the first stage, the MRI image is taken as an input into the detection process. Image pre-processing is a technique to remove the noise from the input image and to gain a perfect image with accurate values as input to proceed with a detection process which eventually ensures accuracy.

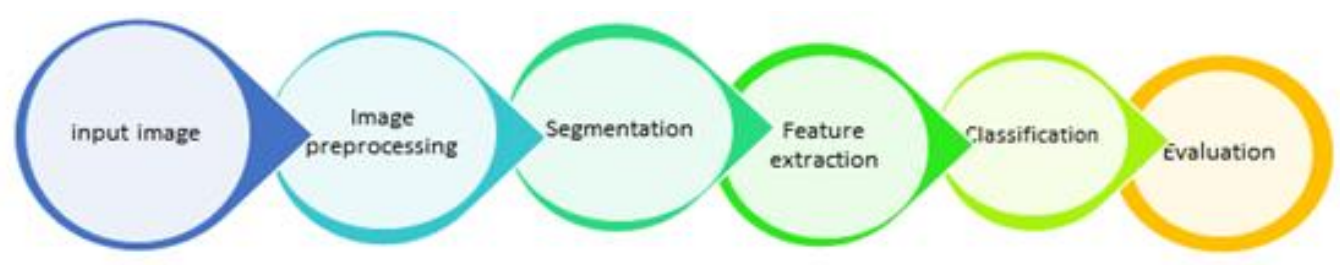

Fig. 1. Stages in bone cancer detection process.

A similar approach from a different field comes from work done by Varvara Nika [10], who has captured local spatial changes between the reference and brain test images. This is done by taking the average of all the absolute differences of a test image block and the blocks found in a local dictionary (best approximation blocks) that are trained from neighbouring blocks of the reference image. The components used in this paper are an Eigen Block Change Detection (CD) algorithm via dictionary learning techniques, adaptive dictionary learning, an Eigen-Block dictionary and PCA (principal component analysis) and numerical simulations. This paper proposes an algorithm to perform local image registration which identifies important structural changes such as the appearance of new lesions or the disappearance of existing ones and growth or shrinkage of the lesion. This work uses eigenvectors in detection of changes to a lesion in the brain. This can be utilized in the present improved proposal as it provides accurate results and can, 
therefore, improve bone tumour detection process.

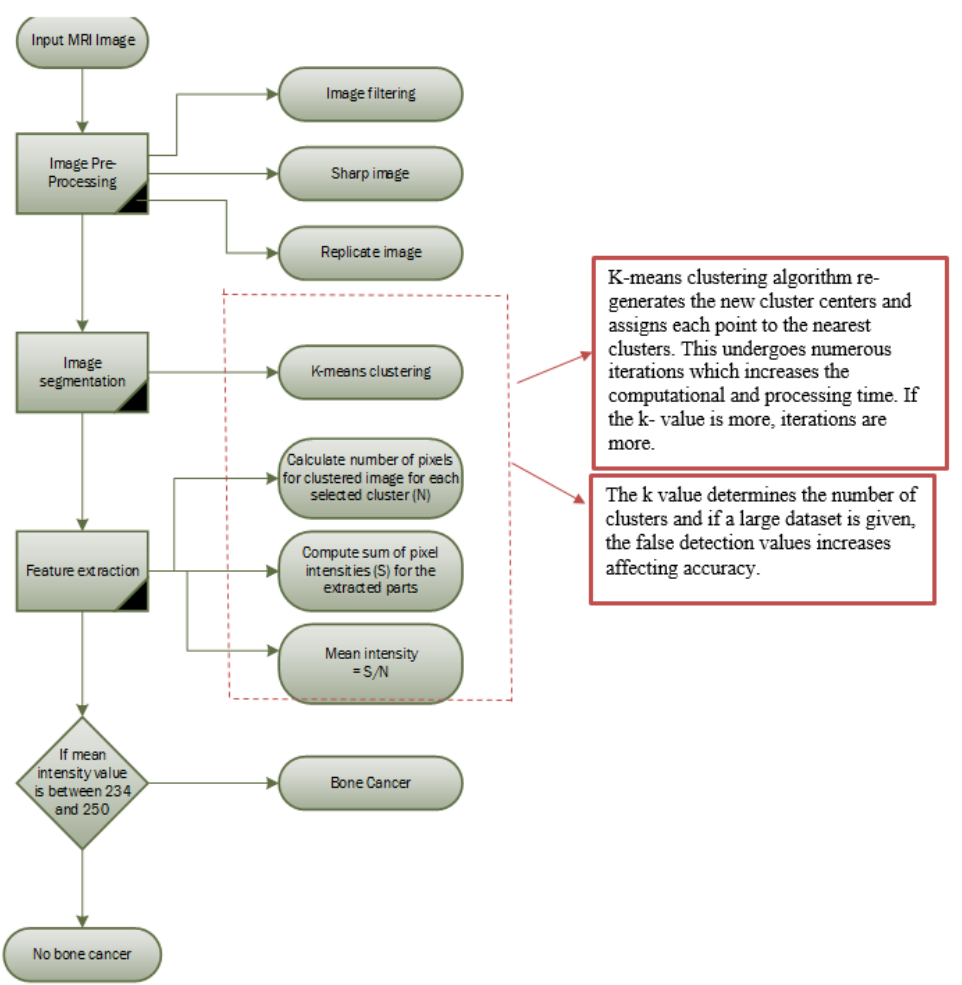

Fig. 2. Flow diagram of current solution highlighting the limitations.

\subsection{Current Best Solution and Limitations}

A detection system proposed by Avula, Lakkakula \& Raja [6] uses clustering. In the clustering process, classification of the pixels depends upon the standardized characteristics. In this research, the algorithms implemented for segmentation process [11], [12] are k-means clustering and seeded region growing. The pixel values will be similar to other clusters in an image which is being segmented into different clusters using k-means clustering algorithm [13]-[15]. It is an iterative process which includes classification of objects into $\mathrm{k}$ number in the group, depending upon the features and attributes. The points are assigned for each cluster which are nearest to the centre, i.e., the centroid which is the average of all the points. Then, arithmetic mean of all the centres is calculated for every dimension [16].

Building non convex shaped clusters and predefinition of the clusters are the main drawbacks of using this k-means algorithm. Mean value could be manipulated with the small change due to noise as the $\mathrm{k}$-means is receptive to noise. Value of $\mathrm{k}$ and the iterations are directly proportional to each other. For a given lower dataset, the results would be better. It is not recommendable for huge datasets. Figure 2 shows the flow of the process and its limitations are high lightened. From the experimental analysis, it is established that the mean pixel intensity values lie in between 234 to 250 .

\section{Proposed Model}

To overcome the limitations of the current solutions, the proposed model uses eigenvectors and eigenvalues while segmenting the image into clusters. Even though the method proposed by Avula, Lakkakula, \& Raja [6] provides 95\% accuracy in detecting bone cancer, there are problems with the number of iterations depending upon the pixel selection which may result in the false detection ([2], [3], [10], [17], [18]. To overcome this, in the proposed methodology, eigenvectors are used for the classification process 
which produces accurate results and also improves the processing time by eliminating iterations. Furthermore, in the proposed model, a simple and effective threshold technique can be applied to the acquired images to reduce the process complexity which helps to improve processing time.

The proposed model is further improved through Shi and Malik's [19] algorithm which solves the segmentation issues using a unique type of eigenvector. Generalized eigenvectors are considered instead of focussing the first eigenvector of $\mathrm{W}$ (image matrix of pixels). Let $\mathrm{D}$ be the degree matrix of $W$ :

$$
D(i, i)=\sum_{\mathrm{j}=0}^{\mathrm{n}} \mathrm{W}(i, j)
$$

The image is cut into two parts by thresholding the second generalized eigenvector of $W$. Minimising the subjects to constraints is the primary goal for the researchers to suggest the second generalized eigenvector which stands as a solution to the discrete problem of segmentation.

The affinity within each group minimizes the affinity between the groups and then normalized which is a segmentation process that overcomes the discrete problem. The second generalized eigenvector provides better information on the precise segmentation although it does not consist two values.

A. Proposed Methodology Algorithm

Step 1: Input image: data set $X=\left\{x_{i}\right\}$ where $1 \leqslant i \leqslant n$. Each $x_{i}$ has d dimensions, so

$X \subset$ Pairwise affinity matrix:

Step 2: Affinity matrix:

$$
W(i, j)=\mathrm{e}-|| x_{i}-x_{j}|| 2 / 2 \sigma 2
$$

where $1 \leqslant i, j \leqslant n$.

Step 3: Degree matrix:

$$
D(i, i)=\operatorname{sum}_{j}(W(i, j))
$$

Step 4: Construct $\mathrm{L}_{\mathrm{ij}}=A_{i j} /\left(D_{i i} D_{j j}\right)^{\wedge} 1 / 2$

This is normalized by row and column degree.

Step 5: Find the $k$ largest eigenvectors of $L$, called $y_{1}, \ldots, y_{k}$.

Form the matrix of column vectors

$X=\left[y_{1}, y_{2} \ldots y_{k}\right] \in$

Step 6: Normalize the rows:

$$
X_{i j}=X_{i j} /\left(\operatorname{sum}_{j}\left(X_{i j}{ }^{2}\right)\right.
$$

Step 7: Average the selected normalized eigenvectors.

The MRI image is taken as the input for the process. Pre-processing techniques are applied to the image for removing the noises. A wiener filter is applied to the image in MATLAB which performs two-dimensional adaptive noise removal filtering. When a constant power adaptive noise degrades the image, this image can be filtered by using Wiener2-lowpass filters. The statistics are estimated from the local territory of every pixel based on which the pixel wise adaptive Wiener method is used by Wiener2. The image is replicated to get all the pixels included in the matrix for calculation without leaving out the first row and first column. All the pixel values are taken into a matrix of rows and columns followed by calculation of the affinity matrix. The affinity matrix is then used to calculate the distance between the pixels in an image.

Affinity matrix is an $N \times N$ matrix with each element $A(i, j)$ the affinity between data point $x_{i}$ and $x_{j}$. The kernel size of these two affinities is problematic. For intensity based affinity, the kernel size $i$ should be small. This is because two data points with large intensity difference should not be in the same cluster. For 
distance-based affinity, the kernel size d should not be too small, because even through two data points are far away, they can still be in same cluster. Therefore, the restriction should be looser for distance affinity.

During the image segmentation process, the calculation of the Degree matrix (2) is crucial for calculating the eigenvalues and eigenvectors [6], [20].

Degree matrix (2):

$$
D(i, i)=\sum_{\mathrm{j}=0}^{\mathrm{n}} W(i, j)
$$

In the above equation (2), $i$ refers to rows and $j$ refers to columns. The sum is stored in a row which gives a diagonal matrix. After calculating the degree matrix, the Laplacian matrix (equation (3)) is calculated which gives results for differences between the degree matrix and the affinity matrix. This Laplacian matrix is normalized which provides the eigenvectors. Normalization of distance affinity and intensity: different data sets have different scales of distance between data points. For an image, the largest distance between two $1000 \times 1000$ data points is $1000 \sqrt{ } 2$, but the largest distance for a $10 \times 10$ image is $10 \sqrt{ } 2$. It is beneficial to normalize the distance to the same scale $[0,1]$, such that one parameter setting (i.e. the value) applies to most images.

Analogous to this, intensity affinity also needs normalization. Therefore, normalization of the eigenvectors (4) is introduced in the proposed model which is implemented on eigenvectors that eliminates the iteration process and minimizes computation complexity.

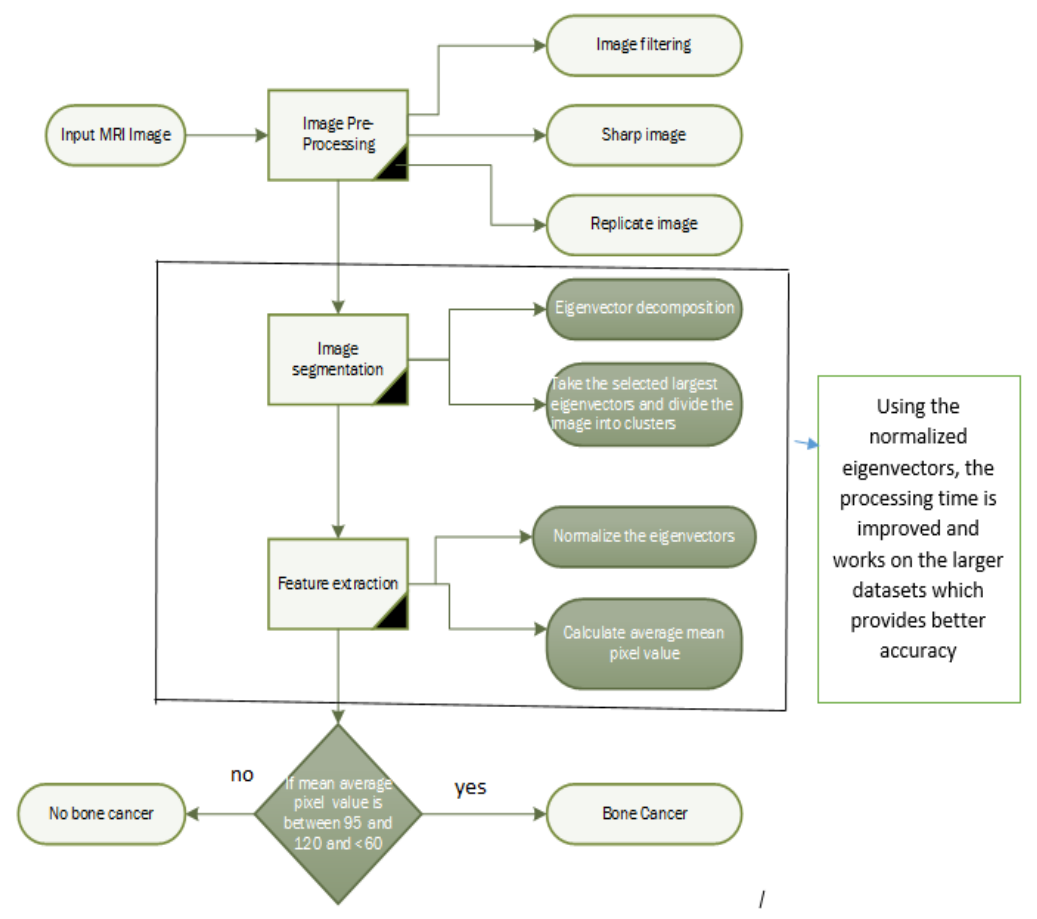

Fig. 3. Flowchart of the proposed method for detecting bone cancer using eigenvectors.

From all eigenvectors derived from Laplacian Matrix, the largest are selected as the higher intensity values are to be taken for processing. These divide the image into clusters and the highest values are selected for forming column vectors which are normalized (equation (4)). The selected normalized eigenvectors are then averaged and the average mean values calculated for the extracted feature.

The experiment is implemented for an average of 60 images in MATLAB R2011a from image processing tool box which includes different types of cancer images and the normal bone MRI images which are not affected by bone cancer. Fig. 3 shows the basic flow of the proposed methodology. After analyzing different 
images, the average mean values calculated on the tumour prone images lies between 95 and 120 and less than 60 . Therefore, if the average mean values match the same, the detection will be true (bone cancer) or else false (no bone cancer).

\section{Discussion and Evaluation}

Bone cancer detection is problematic and remains the subject of intense research activity; it must be identified at the earliest possible stage to enable effective treatment. Experimental results often lead to poor results due to the complexity of the parameters that are involved in the formation of bone cancer. The challenging and the cumbrous part is data mining from MRI images which involves huge datasets consisting MRI images for extracting the required results.

Fig. 4 shows the comparison between the current (using k-means) and proposed methodology (using eigenvectors). The limitations of the current solution and the advantages of the proposed solution are highlighted.

The outputs of the executed methodology are given below. MRI image showing cancer signs are taken and tested accordingly. Each image is divided into different clusters (Fig. 5.1 \& Fig. 5.2) using eigenvectors with the highest eigenvectors utilized as the intensity is higher in the affected areas. The average mean is calculated for the selected clusters and compared (between 95 and 120 which are the outcome of analysis of different images).

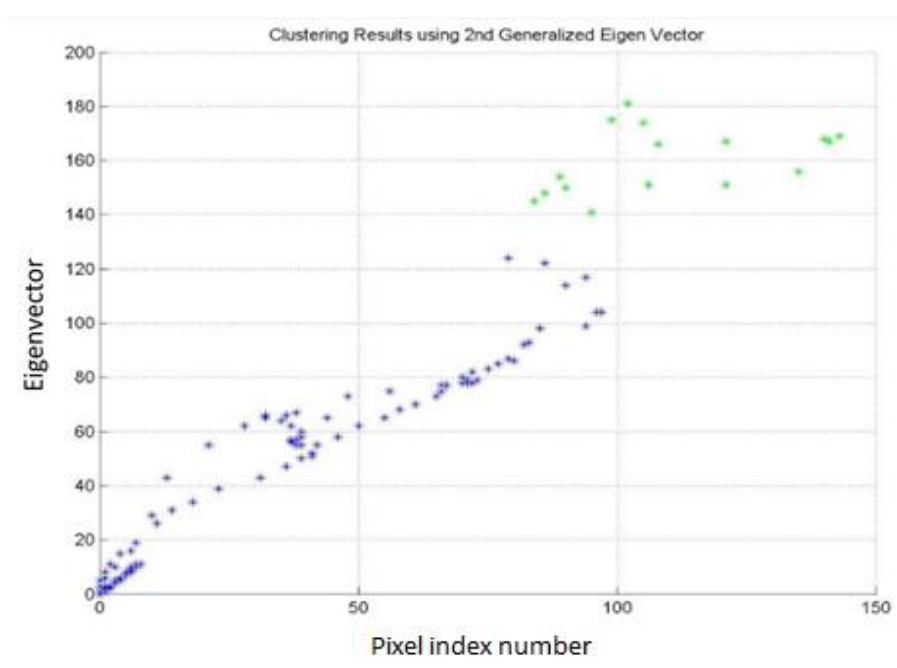

Fig. 4. Clustering the image based on the eigenvectors (largest eigenvectors). Taking the eigenvectors on the $\mathrm{y}$-axis and pixel index number on the $\mathrm{x}$-axis.

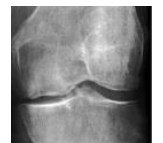

(a)

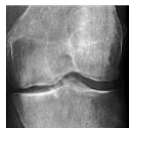

(b)

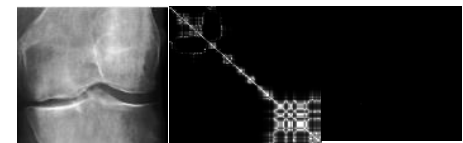

(c) (d) (e)

(a)MRI image (b)Sharp Image (c)Filtered Image (d) Affinity matrix (e) Segmented tumour

Fig. 5.1. Experiment on one of the knee bone MRI image and detection process (cancer not detected).

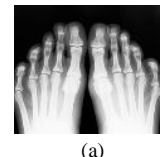

(a)

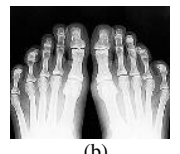

(a)MRI image

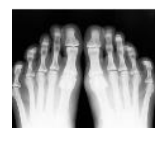

(c)

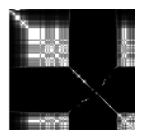

(d)

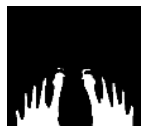

(e)

Fig. 5.2. Experiment on one of the feet bone MRI image and detection process (cancer detected). 
A segmentation process using eigenvectors is applied as shown in Fig. 5.1(a), 5.2(a) and 6(a) respectively to detect the cancer affected parts and segmented images are shown in Fig. 5.1(e), 5.2(e) and 6(e) respectively. Fig. 5.1 (d), 5.2(d) and 6 (d) show the resulting affinity matrix for each image respectively. Fig. 5.1 (b), 5.2(b) and 6 (b) show the obtained sharp image and Fig. 5.1 (c), 5.2(c) and 6 (c) shows the filtered image. This experiment is carried out using MATLAB R2011a with image processing tool kit. This tool box provides the support for different algorithms and methods for segmentation, classification and a widespread environment for data analysis and visualization.

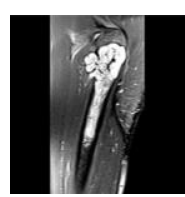

(a)

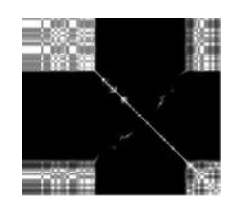

(b)

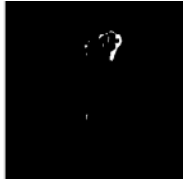

(c)
(a)MRI image
(b) Affinity Matrix
(c) Segmented tumour

Fig. 6. Experiment on the bone MRI image and detection process (cancer detected).

The tumour part is segmented, shown in this process of detection of bone cancer. Depending upon the size of the tumour, the tumour stages and seriousness can be determined. Basically, the experiment is done on different stages of bone tumour images which help in identifying the tumour stage and the level of aggression of bone tumour which helps in processing the treatment of cancer [21]. Fig. 7 shows the difference of the existing method and the proposed method in terms of block diagram.

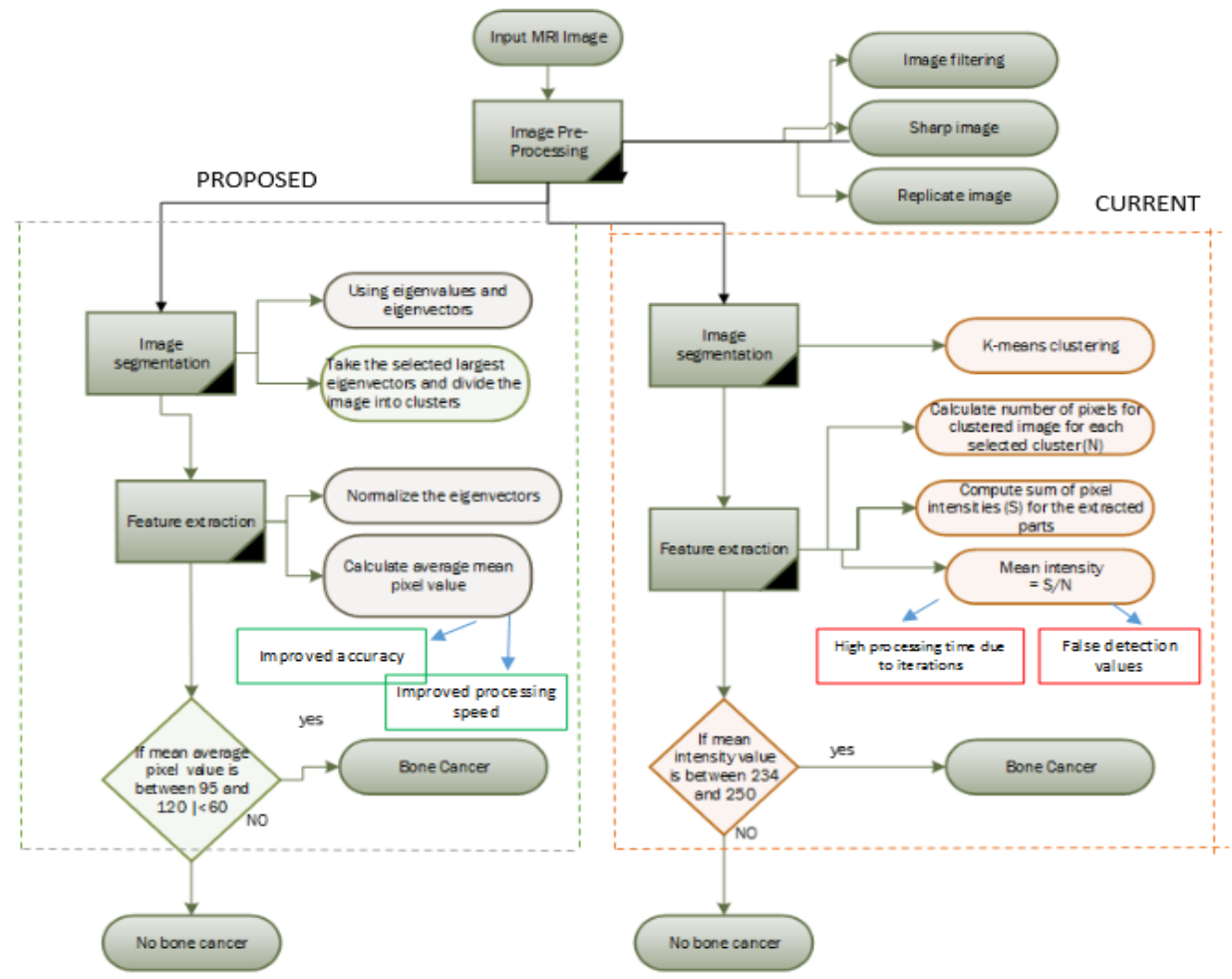

Fig. 7. Differences in the techniques at each step in the bone detection process (current and the proposed solutions).

The following chart (Fig. 8) shows an analysis of computational time needed to analyse different types of bone cancer images including non-cancer images to prove accuracy. Time is logged on the y-axis and 
compares the average processing time between the current and the proposed solutions. From the analysis, it can be said that the proposed method produces superior results to the current method. The number of iterations is higher for the selected number of centres in the current method using k-means meaning that processing time will also be higher when compared to the proposed solution where the largest eigenvectors directly divide images into clusters which does not require iterations and results in lower processing time.

These positive results were also consistent across different age groups as shown in the graph below (Fig. 9).

The proposed solution was performed on general data samples to evaluate the system performance in different scenarios (Table 1). The experiment was performed on 60 images with cancer (Table 2) and without cancer (Table 3). Results between current and proposed methods were shown in the tables. The image set consisted of bones from different parts of the body including knee joints, shoulders, hands, feet etc.

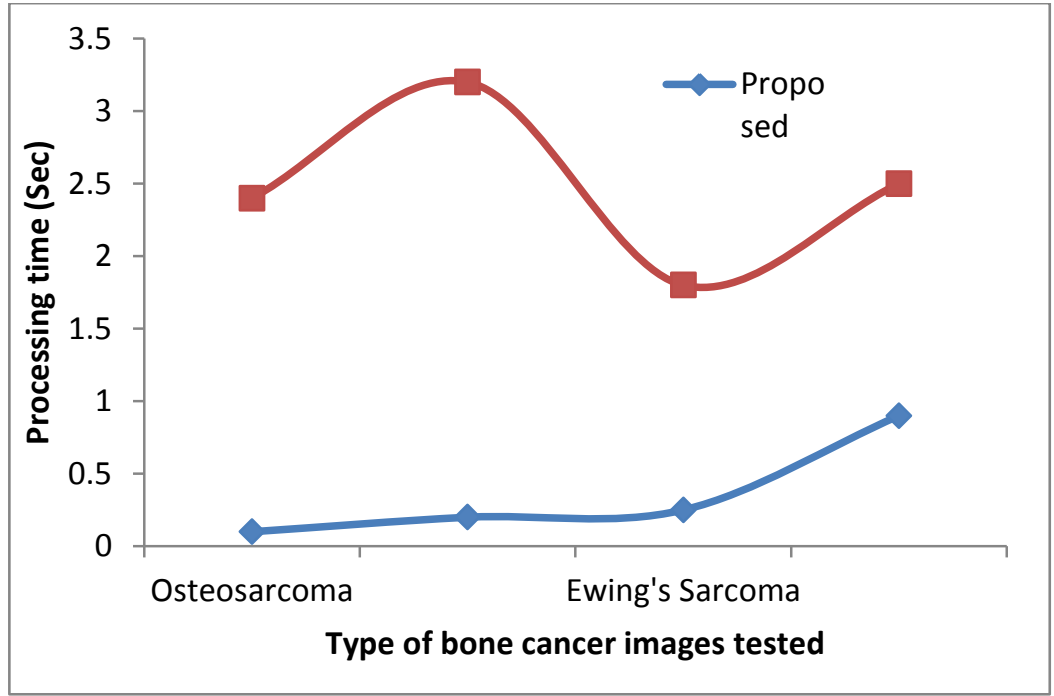

Fig. 8. Average processing time for three different types of bone tumour including no-cancer images taking time on y-axis in seconds.

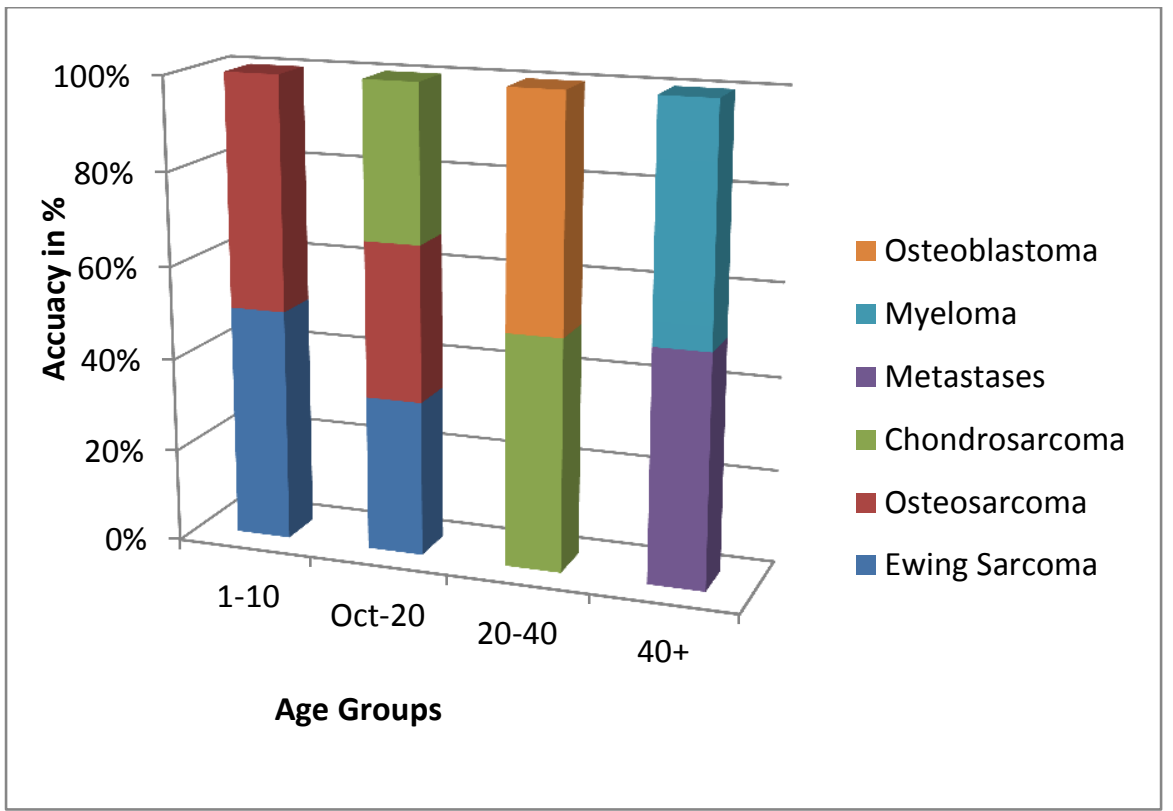

Fig. 9. Accuracy of detection on different types of bone cancers on different age groups. 
Table 1. Experiment on the General Images with the Bone Tumour/ without Tumour

\begin{tabular}{|c|c|c|c|c|c|}
\hline No & $\begin{array}{l}\text { Sample } \\
\text { Image }\end{array}$ & Average & Time & Detection & $\begin{array}{l}\text { Segmented } \\
\text { Tumor }\end{array}$ \\
\hline 1 & & 125.03 & 0.0023 & No & - \\
\hline 2 & & 104.52 & 0.0019 & No & - \\
\hline 3 & & 51.30 & 0.0020 & Yes & \\
\hline 4 & & 73.69 & 0.0018 & No & - \\
\hline 5 & & 124.30 & 0.0020 & No & - \\
\hline 6 & & 121.52 & 0.0019 & Yes & \\
\hline 7 & 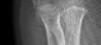 & 69.88 & 0.0023 & Yes & \\
\hline 8 & & 48.97 & 0.0019 & Yes & \\
\hline
\end{tabular}

Table 2. Experiment on the Images with Bone Tumour and Evaluating the Accuracy

\begin{tabular}{|c|c|c|c|c|c|c|c|c|}
\hline \multicolumn{5}{|c|}{ Current method (using k-means) } & \multicolumn{4}{|c|}{ Proposed method (eigenvectors) } \\
\hline Image & Result & Average & Time & Detection & Result & Average & Time & Detection \\
\hline brain1.jpg & no & 190.2 & 0.9026 & FALSE & yes & 98.8784 & 0.21118 & TRUE \\
\hline figure1.jpg & yes & 235.5589 & 1.7124 & TRUE & yes & 103.7614 & 0.027718 & TRUE \\
\hline fr1.jpg & yes & 235.235 & 1.8357 & TRUE & yes & 104.5213 & 0.10173 & TRUE \\
\hline i3.png & yes & 244.2984 & 1.5493 & TRUE & yes & 56.0221 & 0.11074 & TRUE \\
\hline im4.jpg & yes & 234.5386 & 0.6327 & TRUE & yes & 51.3017 & 0.087419 & TRUE \\
\hline image.jpg & yes & 236.885 & 1.9254 & TRUE & yes & 115.3092 & 0.043518 & TRUE \\
\hline image5.jpg & yes & 208.9187 & 0.2876 & TRUE & yes & 57.5729 & 0.22981 & TRUE \\
\hline Y5.jpg & yes & 238.3264 & 1.368 & TRUE & yes & 57.3943 & 0.034463 & TRUE \\
\hline Y1.png & yes & 235.5965 & 1.6349 & TRUE & yes & 56.7512 & 0.11864 & TRUE \\
\hline y2.jpg & yes & 238.506 & 1.5167 & TRUE & yes & 54.8452 & 0.10641 & TRUE \\
\hline y3.jpg & yes & 235.907 & 1.3757 & TRUE & yes & 38.6898 & 0.1073 & TRUE \\
\hline y4.jpg & yes & 245.3932 & 2.8576 & TRUE & yes & 95.049 & 0.24608 & TRUE \\
\hline y7.jpg & yes & 245.23 & 1.3327 & TRUE & yes & 38.8776 & 0.093027 & TRUE \\
\hline y8.jpg & no & 232.5736 & 3.0672 & FALSE & yes & 44.9524 & 0.037028 & TRUE \\
\hline y9.jpg & yes & 240.7453 & 1.7179 & TRUE & yes & 59.5927 & 0.029329 & TRUE \\
\hline Y10.jpg & yes & 237.44 & 1.5648 & TRUE & no & 68.0931 & 0.11036 & False \\
\hline
\end{tabular}


The experiment was also performed on the images without cancer (i.e. bone fracture etc.) so that accuracy could be validated. The following table shows the results and true-false detection values including the speed of computation. The types of bone cancer examined were Osteosarcoma, Chondrosarcoma and Ewing's sarcoma.

There is one false detection in the current experiment on one cancer prone image which can be seen in Table 1. However, the experiment resulted in true detection values for all other images (cancer prone/ no cancer images) used for the experiment which proves that the accuracy is improved including the processing speed through the proposed model. Table 4 shows the results of the experiment on the tumours affecting different age groups and also depicts the accuracy of detection accordingly.

Table 3. Experiment on the Images without Bone Tumour and Evaluating the Accuracy

\begin{tabular}{|c|c|c|c|c|c|c|c|c|}
\hline \multicolumn{5}{|c|}{ Current method (using k-means) } & \multicolumn{4}{|c|}{ Proposed method (eigenvectors) } \\
\hline Image & Result & Average & Time & Detection & Result & Average & Time & Detection \\
\hline f1.jpg & no & 194.8088 & 1.0761 & TRUE & no & 124.3833 & 0.10603 & TRUE \\
\hline f5.jpg & no & 180.3625 & 1.8853 & TRUE & no & 68.5333 & 0.12196 & TRUE \\
\hline fig1.jpg & no & 254.2105 & 1.9581 & TRUE & no & 200.1372 & 0.22351 & TRUE \\
\hline figure2.jpg & no & 203.6937 & 0.2993 & TRUE & no & 90.4139 & 0.031366 & TRUE \\
\hline footxray.jpg & no & 221.333 & 0.4862 & TRUE & no & 125.0339 & 0.10743 & TRUE \\
\hline im1.jpg & no & 203.164 & 1.5167 & TRUE & no & 61.4323 & 1.3166 & TRUE \\
\hline im2.jpg & no & 215.7857 & 1.1185 & TRUE & no & 73.6939 & 0.16104 & TRUE \\
\hline im3.jpg & no & 194.9709 & 0.8572 & TRUE & no & 124.3011 & 0.017041 & TRUE \\
\hline image2.jpg & no & 191.7985 & 0.8592 & TRUE & no & 67.0552 & 0.06749 & TRUE \\
\hline image4.jpg & no & 176.2698 & 1.6879 & TRUE & no & 66.9999 & 0.1622 & TRUE \\
\hline n1.jpg & yes & 235.256 & 1.5603 & FALSE & no & 89.5566 & 0.13028 & TRUE \\
\hline n10.jpg & no & 180.3057 & 2.3131 & TRUE & no & 69.885 & 0.1605 & TRUE \\
\hline n11.jpg & no & 233.5566 & 1.0402 & TRUE & no & 87.304 & 0.11886 & TRUE \\
\hline n2.jpg & no & 251.93 & 0.9257 & TRUE & no & 62.713 & 0.10523 & TRUE \\
\hline n5.jpg & no & 165.235 & 1.4015 & TRUE & no & 129.9572 & 0.045439 & TRUE \\
\hline n6.jpg & no & 178.235 & 1.0597 & TRUE & no & 121.5264 & 0.064335 & TRUE \\
\hline n7.jpg & no & 163.0874 & 4.614 & TRUE & no & 121.3165 & 3.7502 & TRUE \\
\hline n8.jpg & no & 195.235 & 1.5084 & TRUE & no & 135.0736 & 0.085128 & TRUE \\
\hline n9.jpg & no & 226.8664 & 1.5201 & TRUE & no & 132.1029 & 0.092849 & TRUE \\
\hline y10.jpg & yes & 237.4456 & 1.5648 & TRUE & no & 59.0931 & 0.11036 & TRUE \\
\hline
\end{tabular}

Table 4. Experiment on Different Age Groups and Bone Tumour Detection Accuracy

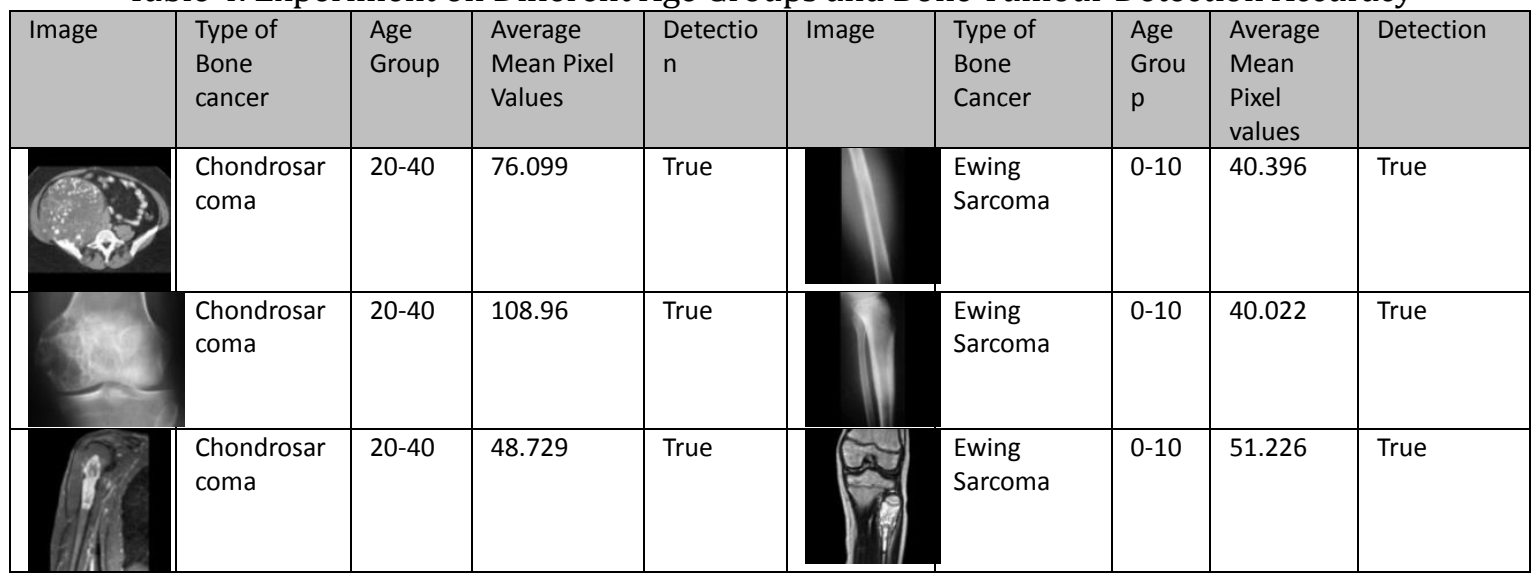




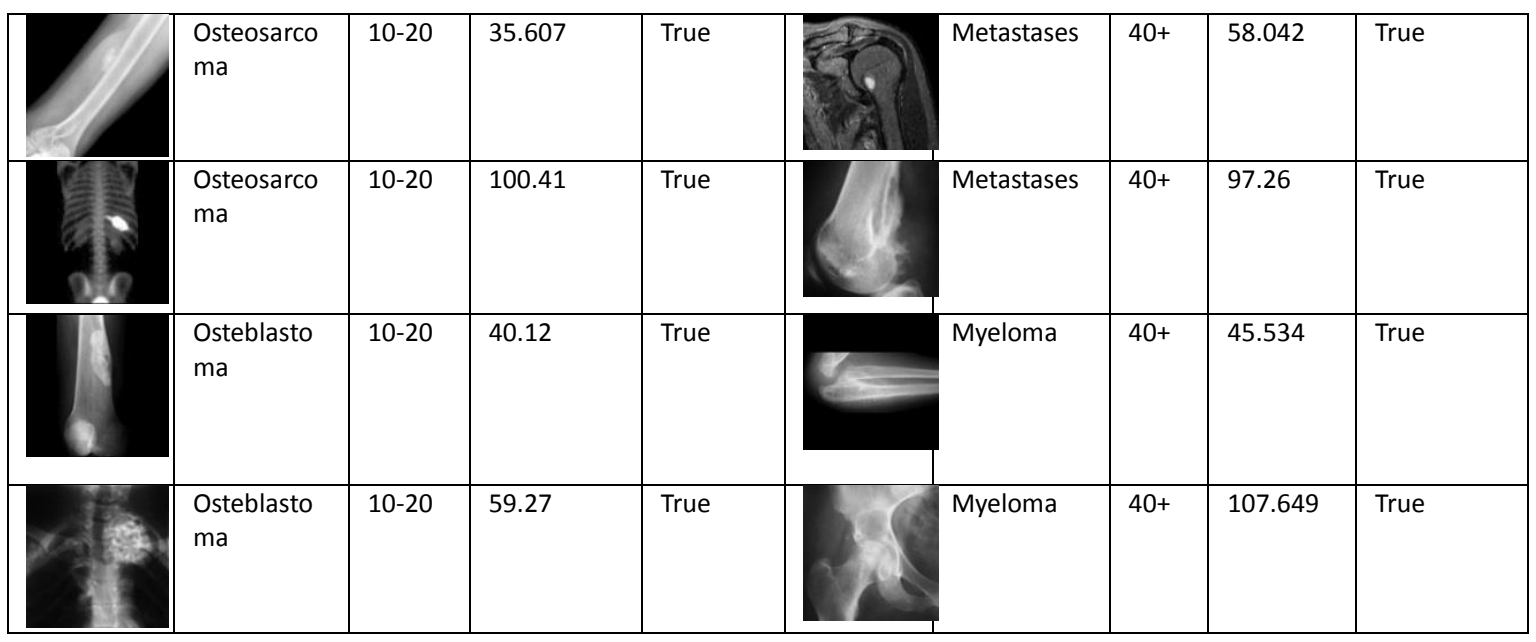

\section{Conclusion}

This paper presents a thresholding technique for choosing the highest eigenvectors (after decomposing images into clusters) and calculating the mean average values to discriminate between cancer/no cancer. An approach to segmenting a tumour or cancer part from an image is implemented using eigenvector decomposition for the extraction of the bone tumour part. The extracted segmented image is further processed to evaluate the mean average in the selected clusters (region of interest). With the mean average value threshold known, higher accuracy rates are achieved in the, detection of bone cancer. The proposed technique achieves a higher rate of accuracy and improves the speed (processing time) with which the tumour is detected. The limitations of the proposed work lies in the possibility of false value detection when performed on the broken bone images. Further studies focus on using eigenvector decomposition techniques in different types of cancers of the brain, spine etc. and also attempt implementations on bone lesions.

\section{References}

[1] Abdellatif, T. Z. (2013). Automatic pectoral muscle boundary detection in mammograms. Proceedings of the 29th National Radio Science Conference (pp. 633-640).

[2] Achilleas, F. H. (1999). Segmentation of biomedical images with eigenvectors. IEEE Trans, 90-93.

[3] Guohui, M. D. (1999). Minimum component eigen-vector based classification technique with application to TM images. Proceedings of Acoustics, Speech, and 1999 IEEE International Conference on Signal Processing (pp. 1520-6149).

[4] Reddy, K. K., Anisha, P. R., \& Narasimha, L. V. (2016). A novel approach for detecting the bone cancer and its stage based on mean intensity and tumor size. Recent Researches in Applied Computer Science, 162-177.

[5] Zhai, H., Huang, H., He, S., \& Liu, W. (2014). Rice Paper classification study based on signal processing. Proceedings of IEEE/ACIS 13th International Conference on Computer and Information Science (ICIS).

[6] Avula, M., Lakkakula, N. P., \& Raja, M. P. (2014). Bone cancer detection from mri scan imagery using mean pixel intensity. Proceedings of 8th Asia Modelling Symposium (pp.141-146).

[7] Chuah, T. K., Reeth, E. V., Sheah, K., \& Poh, C. L. (2013). Texture analysis of bone marrow in knee MRI for classification of subjects with bone. Magnetic Resonance Imaging, 31(7), 930-938.

[8] Urdaneta, M., \& Wahid, P. (2012). A study of UWB imaging for bone cancer detection. Proceedings of International Conference on Ultra-Wideband (pp. 197-201).

[9] Calum, T. C. (2011). Magnetic resonance imaging with k-means clustering objectively measures whole 
muscle volume compartments in sarcopenia/cancer cachexia. Clinical Nutrition, 106-111.

[10] Varvara Nika, P. B. (2014). Eigen block algorithm for change detection - An application of adaptive dictionary learning techniques. Journal of Computational Science, 5(3), 527-535.

[11] Chucherd, S., \& Stanislav S. M. (2011). Sparse phase portrait analysis for preprocessing and segmentation of ultrasound images of breast cancer. IAENG International Journal of Computer Science, 38(2).

[12] Kyaw, M. M. (2013). Pre-segmentation for the computer aided diagnosis system. International Journal of Computer Science \& Information Technology (IJCSIT), 5(1), 79-85.

[13] Rohit, S., \& Gaikwad, M. S. (2013). Segmentation of brain tumour and its area calculation in brain MR images using k-mean clustering and fuzzy C-mean algorithm. International Journal of Computer Science \& Engineering Technology, 524-531.

[14] Bandyopadhyay, S. K., \& Paul, T. U. (2013). Segmentation of brain tumour from MRI image analysis of k-means and DBSCAN clustering. International Journal of Research in Engineering and Science, 77-98.

[15] Fear, X. L. (2002). Confocal microwave imaging for breast cancer detection: Localization of tumors in three dimensions. Biomedical Engineering, 49(8), 812-822.

[16] Khobragade, S., \& Mor, D. (2015). Patil detection of leukemia in microscopic white blood. International Conference on Information Processing (ICIP).

[17] Kourou, K., Exarchos, T., Exarchos, K. E., Karamouzis, M., \& Fotiadis, D. (2015). Machine learning applications in cancer prognosis and prediction. Computational and Structural Biotechnology Journal, 8-17.

[18] Jose, S. R., \& Sambath, M. (2014). Brain tumor segmentation using K-means clustering and fuzzy CMeans algorithms and its area calculation. International Journal of Innovative Research in Computer and Communication Engineering, 2(3), 3496-3501.

[19] Shi, J., \& Malik, J. (2000). Normalized cuts and image segmentation. IEEE TPAMI, 22, 888-905.

[20] Jayadevappa, D., Srinivas Kumar, S., \& Murty, D. S. (2009). A new deformable model based on level sets for medical image segmentation. IAENG International Journal of Computer Science, 36(3), 1-9.

[21] Costelloe, C. M., Hubert, H., Chuang, H., Beth, A., Chasen, T. P., Patricia, S., \& Bassett, R. L. (2013). Bone windows for distinguishing malignant from benign primary bone tumors on FDG PET/CT. Journal of Cancer, 4(7), 524-530.

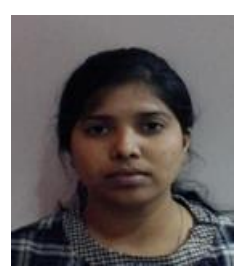

Sravanthi Vallaboju has received his master of information technology, majoring in network security from Charles Sturt University in 2016. She also completed her bachelors in computer science engineering from Geetanjali College India. Her research interests are in the areas of network security and cancer detection. She is currently working on a research project on Leukaemia detection process. She is working as a systems administrator in one of the leading IT company in Australia.

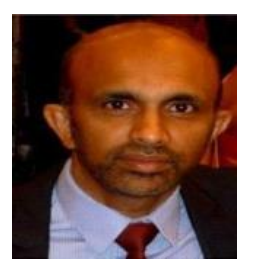

P. W. C. Prasad is an associate professor with the School of Computing and Mathematics at Charles Sturt University, Australia. Prior to this, he was a lecturer at the United Arab Emirates University in UAE, Multimedia University in Malaysia and also the Informatics Institute of Technology (IIT), Sri Lanka. He gained his undergraduate and postgraduate degrees from St Petersburg State Electrotechnical University in the early 90s and completed his Ph.D studies at the Multimedia University in Malaysia. He is an active researcher in the areas of computer architecture, digital systems, and modeling and simulation". He has published more than 130 research articles in computing and engineering journals and conferences proceedings. He has co-authored 
two books entitled 'Digital Systems Fundamentals' and 'Computer Systems Organization and Architecture' published by Prentice Hall. He is a senior member of the IEEE Computer Society.

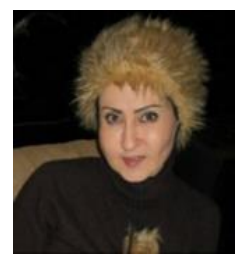

Abeer Alsadoon has academic experience includes working as an associate IT course coordinator and IT lecturer at Charles Sturt University (CSU), Sydney (2012 - up to now), researcher in e-health research group in CSU, Bathurst. She gained her post-doctorate from University of Technology, Sydney (UTS). She received her Ph.D and master studies from the University of Technology, Baghdad in Iraq. She is holding a certified associate project manager (CAPM) from Canada. Abeer's Biography is available in "Who's Who in the World " book that is published in America. It includes the recognized people Biographies in the world. She has significant passion in research and teaching. Alsadoon has published more than 65 IEEE conferences and 7 journal papers level Q1 \& Q2, mainly in medical engineering, e-health, and bioinformatics.

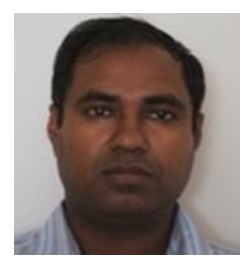

Manoranjan Paul received B.Sc Eng. (hons.) degree in computer science and engineering from Bangladesh University of Engineering and Technology (BUET), Bangladesh, in 1997 and Ph.D degree from Monash University, Australia in 2005. He was a post-doctoral research fellow in the University of New South Wales from 2005 to 2006, Monash University from 2006 to 2009, and Nanyang Technological University from 2009 to 2011. Currently he is an associate professor, steering committee member of the data science research unit, and head of e-health research group at CSU.

His major research interests are in the field of data science such as big data compression, video technology, computer vision, e-health, hyperspectral imaging, and medical imaging. He has published more than 135 refereed publications. He was an invited keynote speaker in CWCN 2017, IEEE WoWMoM 2014, IEEE DICTA 2013, and IEEE ICCIT 2010. Paul is a senior member of the IEEE and ACS. He has served as a guest editor of five issues of journal of multimedia and journal of computers. Currently, Paul is an associate editor of EURASIP journal on advances in signal processing. He was a program chair of PSIVT 2017 and publicity chair of IEEE DICTA 2016.

He obtained research excellence award 2013 and research excellence supervision award 2015 at Faculty level. He also obtained research excellence awards 2017 and 2011 at school level at CSU. He obtained more than $\$ 1 \mathrm{M}$ competitive grant money including the most prestigious Australian research council (ARC) discovery project grant. More information can be found http://csusap.csu.edu.au/ rpaul/.

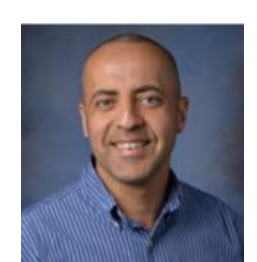

Amr Elchouemi holds a Ph.D in computer science from the University of Louisiana at Lafayette. Elchouemi also hold two masters degrees in computer science and a bachelor degree in computer science as well. Elchouemi has over 25 years of industrial experience in companies like Intel, AMD, and HP. He holds multiple certifications for e-business consulting and networking. His areas of research and experience include computer architecture, cloud computing, e-learning, and computer networks. 